\title{
Spontaneous generation of bending waves in isolated Milky Way-like discs
}

\author{
Matthew H. Chequers ${ }^{\star}$ and Lawrence M. Widrow $†$ \\ Department of Physics, Engineering Physics \& Astronomy, Queen's University, Kingston, ON K7L 3N6, Canada
}

Accepted XXX. Received YYY; in original form ZZZ

\begin{abstract}
We study the spontaneous generation and evolution of bending waves in $N$-body simulations of two isolated Milky Way-like galaxy models. The models differ by their disc-to-halo mass ratios, and hence by their susceptibility to the formation of a bar and spiral structure. Seeded from shot noise in the particle distribution, bending waves rapidly form in both models and persist for many billions of years. Waves at intermediate radii manifest as corrugated structures in vertical position and velocity that are tightly wound, morphologically leading, and dominated by the $m=1$ azimuthal Fourier component. A spectral analysis of the waves suggests they are a superposition of modes from two continuous branches in the Galactocentric radius-rotational frequency plane. The lower-frequency branch is dominant and is responsible for the corrugated, leading, and warped structure. Over time, power in this branch migrates outward, lending credence to an inside-out formation scenario for the warp. Our power spectra qualitatively agree with results from linear perturbation theory and a WKB analysis, both of which include self-gravity. Thus, we conclude that the waves in our simulations are self-gravitating and not purely kinematic. These waves are reminiscent of the wave-like pattern recently found in Galactic star counts from the Sloan Digital Sky Survey and smoothly transition to a warp near the disc's edge. Velocity measurements from Gaia data will be instrumental in testing the true wave nature of the corrugations. We also compile a list of "minimum requirements" needed to observe bending waves in external galaxies.
\end{abstract}

Key words: galaxies: kinematics and dynamics - galaxies: evolution - galaxies: structure - Galaxy: disc

\section{INTRODUCTION}

It is common for disc galaxies to bend in and out of their respective midplanes. The most conspicuous examples are the warps seen in the outer regions of both $\mathrm{HI}$ and stellar discs (see Binney 1992 and Sellwood 2013, and references therein). There are classic "integral sign" warps as well as warps with more complicated morphologies. Indeed, detailed H I maps of the Milky Way reveal a complex pattern of warping and flaring beyond the Solar circle (Levine et al. 2006). More recently, Reylé et al. (2009) showed that warped distortions similar to that in the gas also exist in the stellar and dust components of the Milky Way (see also Cox et al. 1996 for an earlier discussion of warps in the stellar and gaseous components of external discs).

Warps are but one example of how a galactic disc might

^ E-mail: 12mhc@queensu.ca

$\dagger$ E-mail: widrow@queensu.ca deviate from planarity. For example, a disc may also exhibit corrugations or wave-like structures in the direction normal to its midplane. Such structures are most easily observed in the Milky Way where we have access to the full six-dimensional phase space. Consider the Monoceros ring, an overdensity in stars that arcs around the Galactic centre at a Galactocentric radius of about $18 \mathrm{kpc}$ (Newberg et al. 2002; Yanny et al. 2003; Li et al. 2012; Morganson et al. 2016). The Monoceros ring was originally thought to comprise tidal debris from a disrupted dwarf galaxy (Martin et al. 2004; Peñarrubia et al. 2005). The alternative is that it is a feature intrinsic to the disc. For example, several authors have pointed out that the Monoceros ring arose from warping and flaring of the disc (Momany et al. 2004, 2006; Hammersley \& López-Corredoira 2011) or resulted from the disc interacting with a massive satellite (Kazantzidis et al. 2008; Younger et al. 2008; Purcell et al. 2011; Gómez et al. 2016). More recently, $\mathrm{Xu}$ et al. (2015) suggested that the Mono- 


\section{Matthew H. Chequers and Lawrence M. Widrow}

ceros ring is one of several crests of an oscillatory bending wave observed in the direction of the Galactic anti-centre.

The counterpart to these Galactic waves may have been observed in external galaxies. For example, corrugations in the line-of-sight velocity field have been detected in the $\mathrm{H} \alpha$ emission of nearly face-on spiral galaxies by Alfaro et al. (2001) and Sánchez-Gil et al. (2015). These wave-like variations in the vertical velocity field appear to coincide with large amplitude spiral arms. One explanation is that they arise from the interaction between spiral density waves and the galaxy's gaseous disc via the hydraulic bore mechanism (Martos \& Cox 1998; Martos et al. 1999). On the other hand, simulations show that corrugated velocity patterns in the vertical velocity field arise in both stellar and gaseous discs (Gómez et al. 2016, 2017), a result that suggests a gravitational rather than hydrodynamic origin.

Departures from planarity in the disc of the Milky Way often appear as asymmetries in the phase space distribution of stars North and South of the Galactic midplane. For example, the discovery of bending waves by Xu et al. (2015) involved mapping the North-South number count asymmetry as a function of position in the disc plane. Evidence for a North-South asymmetry in the vertical profile of number counts for a $1 \mathrm{kpc}$ cylinder centered on the Sun had already been found by Widrow et al. (2012) and Yanny \& Gardner (2013) (see Ferguson et al. 2017 for a more recent confirmation using a larger photometric sample). Furthermore, Widrow et al. (2012), Williams et al. (2013), Carlin et al. (2013), and Sun et al. (2015) observed bulk motions in Solar Neighbourhood disc stars perpendicular to the midplane (vertical motions). These motions can be interpreted as a combination of the bending and breathing modes that are theoretically predicted for a plane-symmetric system (Mathur 1990; Weinberg 1991; Gómez et al. 2013; Widrow et al. 2014; Widrow \& Bonner 2015; Gómez et al. 2016, 2017).

The existence of warps has been attributed to the tidal effects of satellite galaxies, interactions of the disc with its dark halo, internal excitation of bending instabilities in gaseous discs, and intergalactic winds and magnetic fields, to name just a few (Kahn \& Woltjer 1959; Hunter \& Toomre 1969; Sparke 1984; Sparke \& Casertano 1988; Battaner et al. 1990; Binney 1992; Debattista \& Sellwood 1999; LópezCorredoira et al. 2002; Revaz \& Pfenniger 2004; Shen \& Sellwood 2006). Satellites and halo substructure have been invoked to explain the bending and breathing waves seen in the Milky Way (Widrow et al. 2012; Gómez et al. 2013; Widrow et al. 2014; Feldmann \& Spolyar 2015; D'Onghia et al. 2016; Gómez et al. 2016, 2017), though these features can also be generated by spiral structure (Debattista 2014; Faure et al. 2014; Monari et al. 2016a), the bar (Monari et al. 2015), or some (non-linear) combination of the two (Monari et al. 2016b).

The conclusion is that a galactic disc continually experiences perturbations that can set up waves perpendicular to its midplane. The question remains as to what happens to these waves once they are produced. Are they long-lived or do they quickly decohere, heating and thickening the disc? Can the bending waves be understood as purely kinematic structure, as has been suggested by de la Vega et al. (2015), or is self-gravity essential to their evolution? Finally, is there a connection between the corrugations of $\mathrm{Xu}$ et al. (2015) and the bulk motions in the Solar neighbourhood on the one hand, and the warp at the edge of the Galactic disc on the other?

In this paper, we address these and other questions through a series of idealized isolated galaxy simulations of Milky Way-like models, as well as theoretical arguments based on both the eigenmode and WKB analysis of bending waves by Hunter \& Toomre (1969), Sparke (1984), Sparke \& Casertano (1988), and Nelson \& Tremaine (1995). The bending waves in our simulations arise without any provocation apart from the random noise of the particle distributions used to characterize the (smooth) halo, bulge, and disc. We emphasize that our results are specific to Milky Way-like galaxies and may not generalize to other stellar discs. Indeed, there are very thin disc galaxies that show no sign of bending. These galaxies, which may well be low surface brightness galaxies viewed edge-on (Bizyaev et al. 2017), may not be as susceptible to the dynamical mechanisms described in this work as the galaxies considered here.

Attempts to understand the evolution of galactic bending waves in general, and warps in particular, date back over half a century. Much of the discussion parallels efforts to understand spiral structure. In particular, it was recognized early on that in the absence of self-gravity, an initial warp will shear due to differential precession (Kahn \& Woltjer 1959) in a process akin to the winding problem for kinematic spiral structure. However, Lynden-Bell (1965) argued that a self-gravitating disc might support true bending modes, which makes warps long-lived. This idea was pursued in detail by Hunter \& Toomre (1969) who found that in general, an isolated disc supports a continuum of modes and therefore a generic bending perturbation will disperse. For a disc embedded in a spherically symmetric halo, the only discrete "mode" is the trivial zero-frequency tilt of the disc as a whole. If, on the other hand, the disc is embedded in a flattened halo, then the tilt mode is distorted but remains discrete and is therefore a candidate for explaining longlived warps. This idea, first proposed by Dekel \& Shlosman (1983) and Toomre (1983), was studied in detail by Sparke (1984) and Sparke \& Casertano (1988) who treated the disc as a system of concentric rings embedded in the static potential of a flattened halo (see Revaz \& Pfenniger 2001 for a physical justification of this ring model).

One of the main limitations in Sparke (1984) and Sparke \& Casertano (1988) is that the halo is treated as a static potential, whereas live haloes can respond to the time-evolving gravitational field of the disc. In addition, the disc will set up wakes in the halo, which then act back on the disc via dynamical friction (Chandrasekhar 1943). Bertin \& Mark (1980) argued that the halo-disc interaction can actually excite bending waves that are inside their co-rotation radius, though waves outside co-rotation are damped. Their analysis made a number of approximations, most notably that the halo comprised a spatially uniform Maxwellian distribution of particles. Also, they did not consider the halo contribution to the vertical restoring force. Nelson \& Tremaine (1995) reexamined these claims in the context of more realistic halo models and concluded that the halo is more likely to damp rather than excite bending waves. However, this conclusion itself was called into question by Binney et al. (1998) who carried out numerical experiments that incorporated a ring model for the disc into a conventional $N$-body simulation of 
a dark halo. They found that when the disc is initialized to the Sparke-Casertano tilt-mode, the warp rapidly winds up while its energy remains constant or even increases. Binney et al. (1998) interpret these results in terms of time-scales: the inner halo responds to the disc on a time-scale comparable to the precession period and therefore the potential that the disc precesses in will be markedly different from the one assumed in the static-potential analysis of Sparke (1984) and Sparke \& Casertano (1988). They go on to speculate that there will be true modes of the disc-live halo system, qualitatively similar to but quantitatively different from the Sparke-Casertano modes, that nevertheless avoid the rapid damping predicted in Nelson \& Tremaine (1995).

In this work, we show that a Fourier and spectral analysis of bending waves yields a spectrum that is broadly consistent with both the eigenmode and WKB analysis. Moreover, there is a low-frequency branch of modes with little differential precession. Waves along this branch are therefore long-lived and thus candidates for the bending waves seen in the Galaxy.

Our work is based on $\mathrm{N}$-body simulations of two isolated Milky Way-like galaxy models, which are distinguished by the relative contributions of the disc and halo to the rotation curve, and thus by their susceptibility to the formation of a bar. The models and simulations are described in Section 2. In Section 3, we analyse the vertical bending waves that emerge in our simulated galaxies. In particular, we decompose the waves according to their azimuthal symmetry and present a spectral analysis that is reminiscent of the classic studies of density waves (see Sellwood \& Athanassoula 1986). In Section 4 we show that the qualitative features seen in our spectral analysis are in good agreement with an eigenmode analysis of the linear ring model as well as the dispersion relation derived in the WKB approximation. Section 5 gives a brief description of how we might observe vertical bending waves in external galaxies. We discuss our results in Section 6 and conclude in Section 7.

\section{SIMULATIONS}

\subsection{Two Milky Way-like Models}

We generate the initial conditions for our $N$-body simulations using GalaCtiCs (Kuijken \& Dubinski 1995; Widrow et al. 2008), which allows one to construct axisymmetric disc-bulge-halo systems that are in approximate dynamical equilibrium. The phase space distribution function (DF) for the disc in these models, $f_{d}$, is a function of two exact integrals of motion, the energy and the component of the angular momentum about the symmetry axis, and an approximate third integral that corresponds to the vertical energy. By construction, the disc density obtained by integrating $f_{d}$ over all velocities is well-approximated by the function

$$
\rho_{d}(R, z)=\frac{M_{d}}{4 \pi R_{d}^{2} h} e^{-R / R_{d}} \operatorname{sech}^{2}(z / h) C\left(\left(R-R_{t}\right) / \delta R_{t}\right),
$$

where $R$ and $z$ are cylindrical coordinates and $C$ is a truncation function that goes smoothly from unity to zero at $R \simeq R_{t}$ over a width of order $\delta R_{t}$. The radial velocity dispersion is an exponentially decreasing function of $R$ :

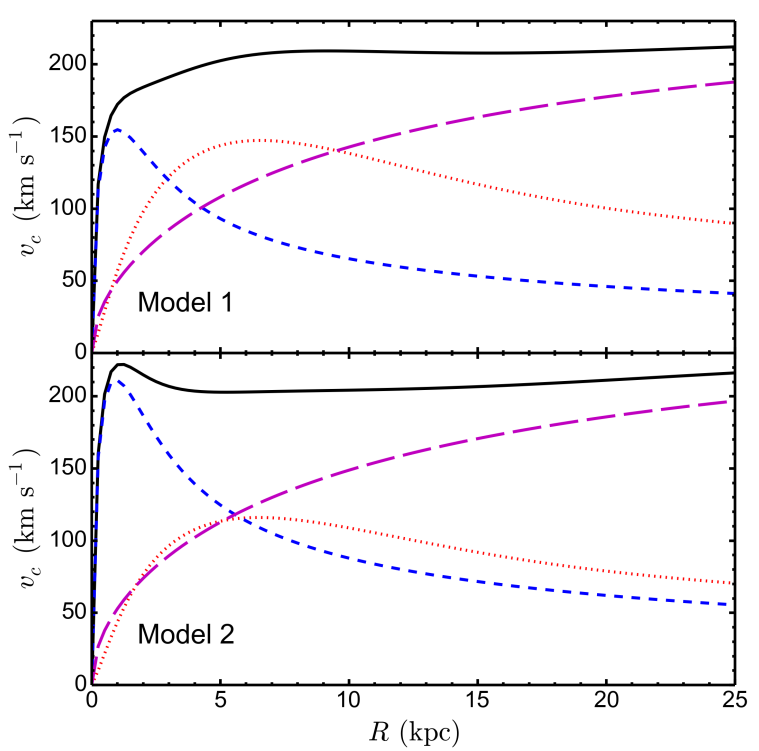

Figure 1. Circular speed curves for the initial conditions of Model 1 (top) and Model 2 (bottom). The solid black curve shows the total circular speed of the model. Also shown are the contributions to the circular speed curve from the bulge (blue, short-dashed), the disc (red, dotted), and the dark matter halo (magenta, long-dashed). Model 1 corresponds to the most stable Milky Way-like model of Widrow et al. (2008), and possesses a disc with enough self-gravity in the inner region to trigger bar formation. Model 2 was constructed with a disc mass $60 \%$ of that in Model 1, while the bulge and halo parameters were adjusted to yield a similar total circular speed curve. Model 2 only forms flocculent spiral structure due to the dominant bulge and halo relative to the disc.

$$
\sigma_{R}(R)=\sigma_{R 0} \exp \left(-R / 2 R_{d}\right),
$$

where we assume that the radial scale length for the square of the velocity dispersion is the same as the scale length for the surface density, in accord with observations (Bottema 1993). The azimuthal velocity dispersion profile is set by the epicycle approximation (see Kuijken \& Dubinski 1995 and Section 3.2.3 of Binney \& Tremaine 2008) while the vertical velocity dispersion is determined from the local surface density and the model assumption that the disc has a constant scale height.

The DFs for the bulge and halo are functions solely of the energy and are therefore isotropic in velocity space. The bulge DF is constructed to yield a density profile that is given, approximately, by

$$
\rho_{b}(r)=\frac{v_{b}^{2}}{4 \pi G R_{e}^{2} c(n)}\left(\frac{r}{R_{e}}\right)^{-p} e^{-b\left(r / R_{e}\right)^{1 / n}},
$$

which yields the Sérsic law for the projected surface density profile with index $n$ so long as one sets $p=1-0.6097 / n+$ $0.05563 / n^{2}$. The constant $b$ is adjusted so that $R_{e}$ encloses half the total projected mass and $c(n)=\left(n b^{n(p-2)}\right) \Gamma(n(2-p))$ (Prugniel \& Simien 1997; Terzić \& Graham 2005). 
Table 1. Initial model parameters.

\begin{tabular}{lcc}
\hline Parameter & Model 1 & Model 2 \\
\hline$M_{d}\left[10^{10} M_{\odot}\right]^{\mathrm{a}}$ & 3.9 & 2.3 \\
$R_{d}[\mathrm{kpc}]^{\mathrm{b}}$ & 2.8 & 2.8 \\
$h[\mathrm{kpc}]^{\mathrm{c}}$ & 0.44 & 0.44 \\
$R_{t}[\mathrm{kpc}]^{\mathrm{d}}$ & 25 & 25 \\
$\delta R_{t}[\mathrm{kpc}]^{\mathrm{e}}$ & 3 & 3 \\
$\sigma_{R 0}\left[\mathrm{~km} \mathrm{~s}^{-1}\right]^{\mathrm{f}}$ & 90 & 56.3 \\
$M_{h}\left[10^{12} M_{\odot}\right]^{\mathrm{g}}$ & 1.2 & 1.3 \\
$v_{h}\left[\mathrm{~km} \mathrm{~s}^{-1}\right]^{\mathrm{h}}$ & 481 & 505 \\
$a_{h}\left[\mathrm{kpc}^{\mathrm{i}}\right.$ & 43 & 43 \\
$M_{b}\left[10^{9} M_{\odot}\right]^{\mathrm{j}}$ & 9.9 & 17.9 \\
$v_{b}\left[\mathrm{~km} \mathrm{~s}{ }^{-1}\right]^{\mathrm{k}}$ & 272 & 375 \\
$R_{e}[\mathrm{kpc}]^{1}$ & 0.64 & 0.64 \\
$n{ }^{\mathrm{m}}$ & 1.32 & 1.32 \\
$Q\left(2.2 R_{d}\right)^{\mathrm{n}}$ & 1.2 & 1.2 \\
\hline \hline
\end{tabular}

Disc mass ${ }^{\mathrm{a}}$; radial disc scale length ${ }^{\mathrm{b}}$; vertical disc scale height ${ }^{c}$; truncation radius of the disc ${ }^{\mathrm{d}}$; truncation width of the disc ${ }^{\mathrm{e}}$; central radial velocity dispersion in the $\operatorname{disc}^{\mathrm{f}}$; halo mass ${ }^{\mathrm{g}}$; characteristic velocity scale of the halo ${ }^{\mathrm{h}} ; \mathrm{NFW}$ halo scale length ${ }^{\mathrm{i}}$; bulge mass ${ }^{\mathrm{j}} ;$ characteristic velocity scale of the bulge ${ }^{\mathrm{k}}$; bulge effective radius ${ }^{\mathrm{l}}$; Sérsic index ${ }^{\mathrm{m}}$; Toomre $Q$ parameter at $2.2 R_{d}{ }^{\mathrm{n}}$.

The halo DF is constructed to yield the NFW profile (Navarro et al. 1996):

$$
\rho_{h}(r)=\frac{a_{h} v_{h}^{2}}{4 \pi G} \frac{1}{r\left(r+a_{h}\right)^{2}} .
$$

While the velocity distributions of the halo and bulge are isotropic, their space densities are slightly flattened due to the disc potential.

In this paper, we consider two GALACTICS models. The first (Model 1) is from Widrow et al. (2008). In that paper, Bayesian and Markov chain Monte Carlo methods were used to constrain the GALACTICS parameters so as to match kinematic and photometric observations of the Milky Way. That analysis yielded a suite of models that varied in their susceptibility to bar formation. The model that we chose for this paper has a relatively weak bar instability. For Model 2, we reduced the disc mass by roughly a third and increased the masses of the bulge and halo components so as to obtain a similar rotation curve. The disc in Model 2 is therefore so light that it never forms a bar.

While our models yield very similar circular speed curves they differ in the relative contributions of the disc and the dynamically hot components (the bulge and halo) to the radial force, as shown in Fig. 1. In particular, Model 1 has a disc mass of $M_{d} \approx 4 \times 10^{10} M_{\odot}$, which impies that at a radius of $2.2 R_{d}, V_{d}^{2} / V_{c}^{2}=0.51$, where $V_{c}=V_{c}(R)$ is the circular speed at radius $R$ and $V_{d}$ is the contribution to the circular speed from the disc. The central radial velocity dispersion is set to $90 \mathrm{~km} \mathrm{~s}^{-1}$, which yields a Toomre $Q$ parameter (Toomre 1964) at $2.2 R_{d}$ of 1.2. For Model $2, V_{d}^{2} / V_{c}^{2}=0.33$ at $2.2 R_{d}$. In addition, the central radial velocity dispersion in the disc is decreased so that the Toomre $Q$ parameter at $2.2 R_{d}$ is the same as in Model 1. The GALACTICS parameters for the two models are given in Table 1.

\section{$2.2 \quad N$-body Simulations}

We use GALACTICS to generate $N$-body realizations for the two models just described. These realizations have $2.5 \mathrm{M}$ disc particles, $250 k$ bulge particles, and $5 M$ halo particles. The masses of particles within each component are identical. The initial conditions were evolved for $\sim 10 \mathrm{Gyr}$ using GADGET-3 (Springel 2005) with a softening length of $40 \mathrm{pc}$ for all particle types. The maximum time step was set to $0.2 \mathrm{Myr}$, which is $\sim 0.2 \%$ of the Galactic dynamical time at a radius of 20 $\mathrm{kpc}$, where the warp occurs, and $\sim 1 \%$ of a Galactic dynamical time at $4 \mathrm{kpc}$. Total energy was conserved to within $0.06 \%$. We start with "pristine" equilibrium initial conditions, which allow us to focus on key dynamical processes such as the secular evolution of bending waves. Our simulations ignore gas dynamics and do not capture the physics of galaxy formation.

Over time the discs in our simulations rotate and drift slightly about the global coordinate origin. To account for this when analyzing face-on maps of our discs we center the disc and rotate it into the $x-y$ plane by way of the following iterative scheme. We compute the center of mass of disc particles within a cylinder of radius $20 \mathrm{kpc}$ and height $2 \mathrm{kpc}$ centered in the $x-y$ plane and translate all disc particles to that frame. This procedure is repeated until a translation of less than $1 \mathrm{pc}$ is achieved in all coordinate directions. Next, we account for any tilt of the disc relative to the $x-y$ plane by using a two-dimensional Newton-Raphson scheme to find the Euler angles about the $x$ - and $y$ - axes that minimize the root mean square vertical displacement of disc particles within a cylinder of radius $20 \mathrm{kpc}$ and height $2 \mathrm{kpc}$ centered in the $x-y$ plane. This method does a good job of diagonalizing the moment of inertia tensor of the disc.

In Fig. 2, we show the disc surface density relative to the equilibrium surface density for the two models at various times. The maps extend to $R=25 \mathrm{kpc}$, or about $9 R_{d}$, which is the radius at which we begin to truncate the disc. This radius also corresponds to the outermost radius at which the warp in the Milky Way's stellar disc has been observed (Momany et al. 2006). The decrease of $\sim 20-55 \%$ in the surface density for $R>22 \mathrm{kpc}$, which develops at early times, is likely due to a relaxation in the initial conditions.

The disc in Model 1 forms a bar at $\sim 2.5 \mathrm{Gyr}$, which eventually extends to $R \sim 5 \mathrm{kpc}$. In addition, a prominent ring (again, an enhancement relative to the initial exponential profile) develops at a radius of $\sim 10-15 \mathrm{kpc}$. This feature is likely a result of mass redistribution due to the bar. On the other hand, the disc in Model 2 forms flocculent spiral structure but no bar.

\section{ANALYSIS OF BENDING WAVES}

\subsection{Fourier Analysis}

The surface density shown in Fig. 2 represents the zeroth moment, with respect to $z$, of the coarse-grained stellar DF, $\tilde{f}:$

$$
\Sigma(R, \phi, t)=\int \tilde{f}(\mathbf{r}, \mathbf{v}, t) d^{3} v d z .
$$

(The true DF for an $N$-body system is a sum of six- 

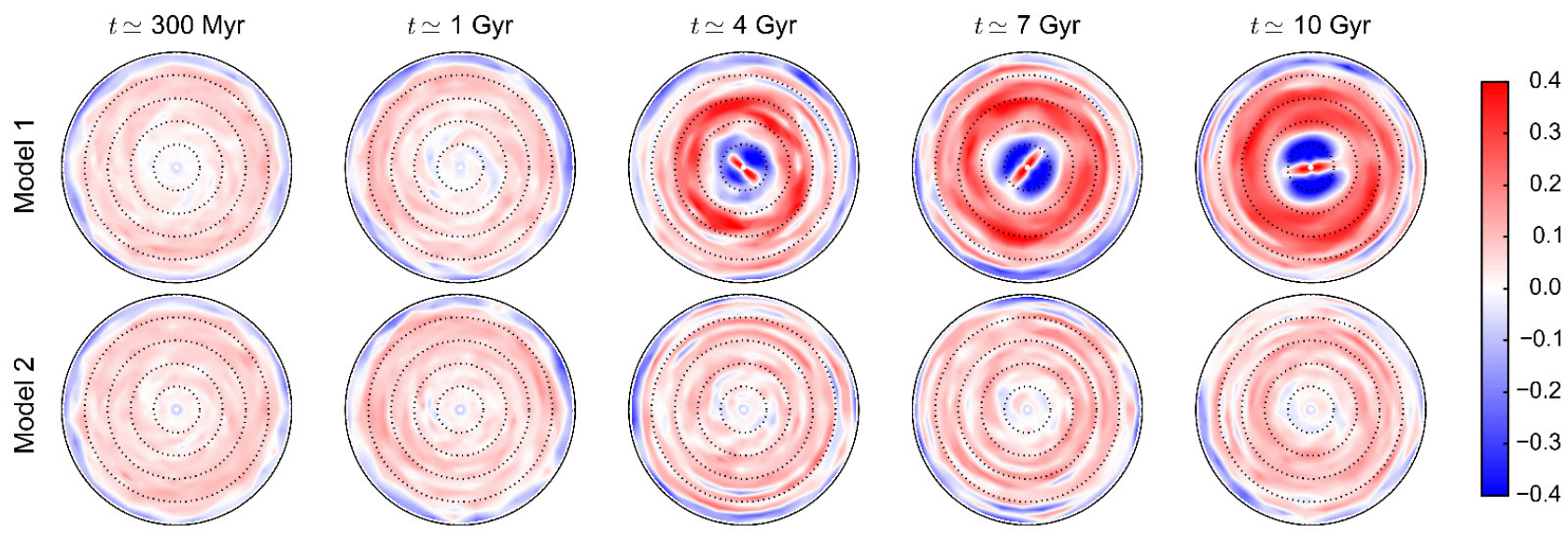

Figure 2. Face-on disc surface density enhancement for Model 1 (top row) and Model 2 (bottom row) at five epochs throughout the simulation, as indicated at the top of each column. The colour map indicates the logarithm (base 10) of the ratio of the surface density at each epoch relative to the initial equilibrium surface density. Dotted concentric circles indicate increments of 5 kpc in radius. The rotation of the discs is counter-clockwise. The bar that forms in the higher mass disc of Model 1 is clearly visible, as well as a ring structure between 10 and $15 \mathrm{kpc}$. Model 2 only forms flocculent tightly wound spiral structure and displays only modest surface density enhancements relative to Model 1.

dimensional $\delta$-functions at the positions of the particles. In practice, coarse-graining amounts to calculating an average surface density over a small patch of the disc.) Likewise, the mean vertical displacement of the disc is given by the first moment with respect to $z$ :

$$
Z(R, \phi, t)=\Sigma(R, \phi, t)^{-1} \int \tilde{f}(\mathbf{r}, \mathbf{v}, t) z d^{3} v d z .
$$

In Fig. 3, we show mean vertical displacement and vertical bulk velocity maps for the two models at the same five epochs shown in Fig. 2. During the first Gyr, displacements of order $100 \mathrm{pc}$ and bulk vertical motions of a few $\mathrm{km} \mathrm{s}^{-1}$ are found across the discs in both models. We interpret these displacements and bulk motions as bending waves. By $4 \mathrm{Gyr}$, the presence of the bar is clearly evident in Model 1. Interestingly enough, by this epoch, the vertical displacements are stronger in Model 2 and extend further in toward the centre of the disc, as compared with Model 1. The implication is that the bar and/or more massive nature of the disc in Model 1 has a damping effect on bending waves.

The waves have a strong $m=1$ component, where $m$ is the azimuthal mode number. Moreover, these waves appear to be leading in the sense that the radius of the peak vertical displacement ridge increases with increasing $\phi$, that is, in the direction of rotation. The $m=1$ waves are tightly wound at intermediate radii but transition smoothly to a warp at the edge of the disc. In general, the amplitude of the waves in the outer disc grows over time while the amplitude decreases in the inner disc. We return to this point below.

The bulk vertical motions follow the same general pattern of the displacements except that they are out of phase, as one would expect for wave-like motion (see Gómez et al. 2013, 2016, 2017). From the amplitudes of the velocity and height perturbations, we infer a characteristic pattern speed for the bending waves of $\sim 10-20 \mathrm{~km} \mathrm{~s}^{-1} \mathrm{kpc}^{-1}$.

In the study of density waves, it has proved instructive to write the surface density as a Fourier series in $\phi$ (see, for example, the seminal work of Sellwood \& Athanassoula 1986). Formally, one has

$$
\Sigma(R, \phi, t)=\operatorname{Re}\left\{\sum_{m} \Sigma_{m}(R, t) e^{-i m \phi}\right\}
$$

where

$$
\Sigma_{m}(R, t)=\frac{1}{2 \pi} \int_{0}^{2 \pi} \Sigma(R, \phi, t) e^{i m \phi} d \phi .
$$

In practice, the $\Sigma_{m}$ are calculated over a ring of finite radius (essentially, coarse graining in $R$ ). For a ring at radius $R_{\alpha}$ and width $\Delta R_{\alpha}$ we have

$$
\Sigma_{m}(R, t)=A_{\alpha}^{-1} \sum_{j \in \alpha} m_{j} e^{i m \phi_{j}}
$$

where $A_{\alpha}=2 \pi R_{\alpha} \Delta R_{\alpha}$ is the area of the ring and the sum is over all particles in the ring. Similar to the surface density, we can construct a Fourier series for the vertical displacement:

$$
Z(R, \phi, t)=\operatorname{Re}\left\{\sum_{m=0}^{\infty} Z_{m}(R, t) e^{-i m \phi}\right\},
$$

where we define

$$
Z_{m}(R, t) \equiv A_{\alpha}^{-1} \sum_{j \in \alpha} \frac{z_{j} m_{j}}{\Sigma\left(R_{j}, \phi_{j}, t\right)} e^{i m \phi_{j}} .
$$

The surface density at particle $j, \Sigma\left(R_{j}, \phi_{j}, t\right)$, is estimated by using the Fourier series for $\Sigma$ (equation 7 ) truncated at the 5 th order terms.

In Fig. 4 we show the $m=0,1,2$, and 3 Fourier modes 

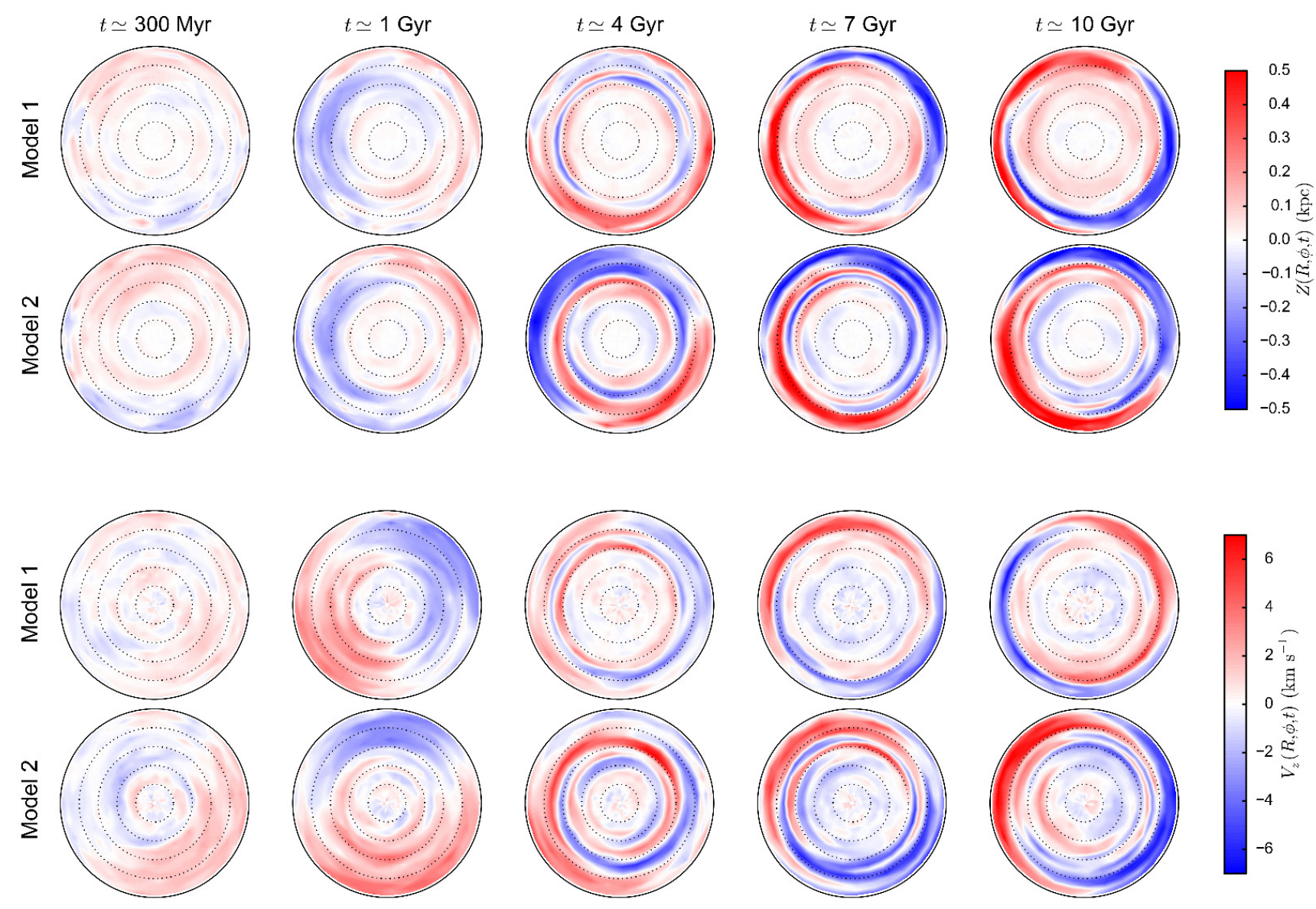

Figure 3. Face-on maps of the mean vertical displacement, $Z(R, \phi, t)$ (top set of rows), and mean vertical velocity, $V_{z}(R, \phi, t)$ (bottom set of rows), for Model 1 and Model 2, as indicated on the left side. Columns correspond to the same five epochs in Fig. 2, as indicated at the top of each column. Dotted concentric circles indicate increments of $5 \mathrm{kpc}$ in radius. The rotation of the discs is counter-clockwise. At later times, the leading nature of the bending waves, especially in the outer disc, is very clear. In general, bending is slightly stronger and more pervasive in the less massive and barless disc of Model 2 compared to Model 1, especially at later epochs. Furthermore, the average height and vertical velocity seem to be coupled, qualitatively similar to the oscillatory anti-correlation previously reported (Gómez et al. 2013, 2016, 2017).

of $Z$ for the $t \sim 4 \mathrm{Gyr}$ snapshot of the Model 1 simulation. We see that while the $m=1$ mode is clearly leading, the $m=2$ term is trailing, and the $m=3$ term is a combination of both leading and trailing. Furthermore, the $m=1$ mode is the dominant mode beyond a radius of $\sim 15 \mathrm{kpc}$.

We explore how this dominant mode in the outer disc forms and evolves in Fig. 5 by showing $\left|Z_{1}(R)\right|$ for both models at various snapshots. Both models show a trend of increasing $\left|Z_{1}(R)\right|$ from intermediate radii to the outer disc with time. This is most evident in Model 1 where there is a factor of $\sim 2-3$ decrease in $\left|Z_{1}\right|$ at $R \sim 10-15 \mathrm{kpc}$ and an approximately equal increase in the outer disc after $t \simeq 1 \mathrm{Gyr}$. We attribute the large suppression of $\left|Z_{1}\right|$ at intermediate radii to the bar, which forms at $\sim 2.5$ Gyr. This trend, which is also present in Model 2 though not so pronounced, suggests that warps may arise from the outward migration of $m=1$ bending waves.

\subsection{Spectral Analysis}

To connect our 3D simulations with the linear analysis of Section 4, we perform a spectral analysis of bending waves in our simulated discs. Our method uses the techniques from Sellwood \& Athanassoula (1986) (see also Roškar et al. 2012 for a succinct description). We calculate the Fourier coefficients $Z_{m}(R, t)$ (see Section 3.1, equation 11) for the $t_{j}=j \Delta_{t}+t_{0}$ snapshots where $\Delta_{t}$ is the time between snapshots, $j=0 \ldots N-1$, and $N$ is even. We then perform a discrete Fourier transform in time to obtain the two-sided spectral coefficients

$$
\mathcal{Z}_{m}\left(R, \omega_{k}\right)=\sum_{j=0}^{N-1} Z_{m}\left(R, t_{j}\right) w(j) e^{2 \pi i j k / N},
$$

(Press et al. 2007, Section 13.4). Here, $w(j)$ is a Gaussian window function with a standard deviation of $N / 2^{5 / 2}$, which is introduced to mitigate spectral leakage from high frequencies. We note that using similarly shaped window functions, such as the Hamming window, does not significantly alter 


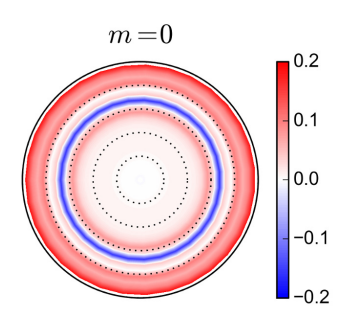

$m=2$

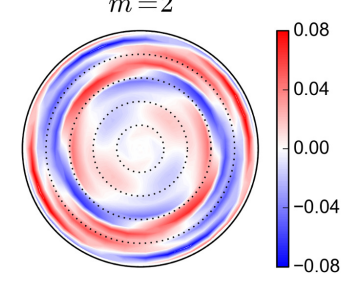

Figure 4. Fourier mode decomposition of $Z(R, \phi, t \sim 4 \mathrm{Gyr})$ for $m=0,1,2$, and 3 , as indicated, for Model 1 . Dotted concentric circles indicate increments of $5 \mathrm{kpc}$ in radius. The rotation of the disc is counter-clockwise. The colour scale is in units of $\mathrm{kpc}$, and differs between the upper and lower panels in order to highlight the extremal values for each $m$. The factor of $1 / 2$ for the zeroth order term in the Fourier Series is reflected in the $m=0$ panel. From the figure it is clear that the (leading) $m=1$ term is the dominant one in the decomposition.

the resulting spectra or our conclusions. The discrete frequencies ${ }^{1}$ are given by

$$
\omega_{k}=\frac{2 \pi}{m} \frac{k}{N \Delta_{t}}
$$

with $k=-N / 2 \ldots N / 2$, and the Nyquist frequency corresponding to $\omega_{k= \pm N / 2}$. The two-sided power spectrum is then computed as

$$
P_{m}\left(R, \omega_{k}\right)=\frac{1}{W}\left|\mathcal{Z}_{m}\left(R, \omega_{k}\right)\right|^{2},
$$

where $W=N \sum_{j=0}^{N-1} w(j)$ is the window function normalization.

In Fig. 6, we explore the time evolution of the $m=1$ bending wave power spectra for Model 1 . To do so, we divide the simulation into five $\sim 2 \mathrm{Gyr}$ intervals and calculate $P_{1}$ using the method outlined above. Each interval has $N=$ 200 snapshots with a time resolution of $\Delta_{t} \approx 9.8 \mathrm{Myr}$, which gives a frequency resolution of $\sim 3.14 \mathrm{~km} \mathrm{~s}^{-1} \mathrm{kpc}^{-1}$. Prominent bands of power develop at early times and persist over the entire simulation. Much of the power lies along two main branches, which, as we describe below, follow the two lowestorder vertical resonances from linear theory. Power along the lower branch, which correponds to slowly counter-rotating

\footnotetext{
${ }^{1}$ Since we consider negative frequencies, we note a difference between our index $k$, and that used in the conventional spectral analysis. For example, the mapping between our $k=-N / 2 \ldots N / 2$ and the index used in Roškar et al. (2012), $k^{\prime}=0 \ldots N-1$, is $k^{\prime}=k$ for $k \geq 0$ and $k^{\prime}=k+N$ for $k<0$. Therefore, the range $k^{\prime}=0 \ldots N / 2-1$ corresponds to increasing positive frequencies, and $k^{\prime}=N / 2+1 \ldots N-1$ corresponds to increasing negative frequencies. The Nyquist frequency for $k^{\prime}=N / 2$ corresponds to $k= \pm N / 2$.
}

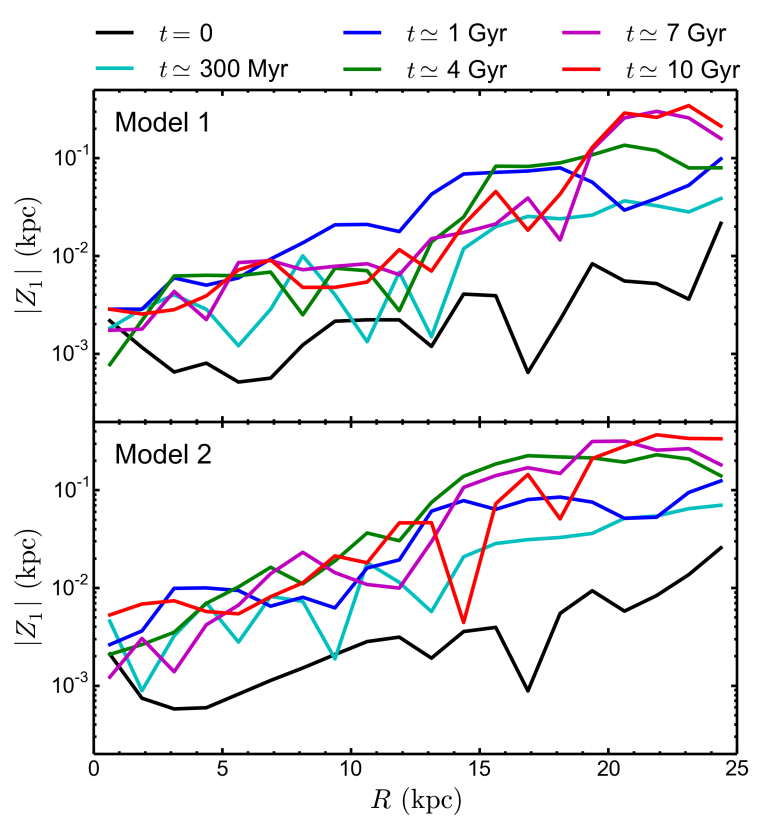

Figure 5. Time evolution of $\left|Z_{1}(R)\right|$. The upper and lower panels show Model 1 and Model 2, respectively. Both models show a general trend of increasing strength propagating outward from intermediate radii to the outer disc over time.

waves, is seen across the disc at all times. On the other hand, power along the upper branch (more rapidly rotating waves) diminshes with time. In general, over time power migrates to larger radii.

We note the presence of spurious bands of power at fixed radii that extend almost the entire frequency range in Fig. 6. More specifically, there is a band present in all time intervals at $R<0.25 \mathrm{kpc}$, as well as weaker bands at larger radii that come and go across the various time intervals. These bands arise when the local surface density term in the vertical displacement Fourier coefficients $\left(\Sigma\left(R_{j}, \phi_{j}\right)\right.$ in equation 11) for one or more particles is computed to be nearly zero. This situation is an artefact of our scheme to estimate the local surface density as a truncated Fourier series.

Overlaid in all panels of Fig. 6 are the $m=1$ vertical resonances (analogous to the Lindblad resonances for density waves), $\omega=\Omega \pm v_{x}$, where $\Omega$ is the circular frequency of the disc and $v_{x}=\sqrt{v_{b}^{2}+v_{h}^{2}}$ is the vertical force oscillation frequency due to the bulge and halo potential, of the initial conditions. Power in the $m=1$ bending waves collects along the vertical resonances and tends to lie outside of the forbidden region between them, as predicted by the WKB approximation (Binney \& Tremaine 2008, Section 6.6.1, but also see Section 4). Furthermore, agreement between our power spectra and the vertical resonances of the initial conditions endures for many billions of years.

We can then ask the question as to why higher frequency waves subside over time, or conversely, why low-frequency waves persist? To this end, we consider a form of the tipLine-of-Nodes (LON) plot (see Briggs 1990) for waves of various pattern speeds. A tip-LON plot is constructed by calculating, for a series of concentric rings, the tilt, or tip 
away from the midplane, of the disc and the position of the LON. To do this, we construct an effective vertical displacement as a function of $R$ and $\phi$ for a narrow range in frequency, $\Delta \omega_{k}$, using only the $m=1$ spectral coefficient of the $t-\omega_{k}$ Fourier transform:

$$
Z_{1}\left(R, \phi, \omega_{k}\right)=\int_{\omega_{k}-\Delta \omega_{k} / 2}^{\omega_{k}+\Delta \omega_{k} / 2} \operatorname{Re}\left\{\mathcal{Z}_{1}\left(R, \omega_{k}\right) e^{i \phi}\right\} d \omega_{k} .
$$

The function $Z_{1}\left(R, \phi, \omega_{k}\right)$ is essentially our series of rings from which the tilt and position angle for the LON as a function of $R$ can be easily determined.

Tip-LON plots are shown in Fig 7. For illustrative purposes we only consider the time interval $2<t<4 \mathrm{Gyr}$ for Model 1 (i.e. Fig. 6, top middle panel) and show the results for $\omega_{k}=28.3,3.1,0$, and $-3.1 \mathrm{~km} \mathrm{~s}^{-1} \mathrm{kpc}^{-1}$. These four frequencies, which are indicated by horizontal dashed lines in Fig. 6, correspond to a typical frequency in the upper branch and three frequencies in the lower branch. Each dot of a tip-LON plot corresponds to a ring within the disc whose radius, tilt, and position angle are encoded in the colour, radial coordinate, and angular position, respectively, of the dot. Thus, dots that trace a clockwise pattern with increasing ring radius indicate a trailing wave.

Large tilt angles for $\omega_{k}=28.3 \mathrm{~km} \mathrm{~s}^{-1} \mathrm{kpc}^{-1}$ are localized to $15 \lesssim R \lesssim 20 \mathrm{kpc}$ where there is a prominent peak in the power spectrum (see Fig. 6). These are clearly trailing waves. On the other hand, the tilt is stronger across a greater range in $R$ at $\omega_{k}=-3.1,0$, and $3.1 \mathrm{~km} \mathrm{~s}^{-1} \mathrm{kpc}^{-1}$. These are leading waves, which experience less shear in the outer disc and therefore persist over a longer period of time.

\section{LINEAR RING MODEL}

In this section, we study the linear bending waves of a razor thin, self-gravitating disc embedded in the static gravitational potential of a bulge and halo. The starting point for this investigation is the second-order integrodifferential equation for local vertical displacements of the disc, $Z(R, \phi, t)$, which was introduced by Hunter \& Toomre (1969). In order to relate this $Z$ to the mean vertical displacement mapped in our simulations, we first show that the equation from Hunter \& Toomre (1969) can be derived from the collisionless Boltzmann equation.

\subsection{Equations of Motion}

We consider a system of collisionless "stars" whose equilibrium distribution is that of a self-gravitating disc embedded in the external potential of an extended dark matter halo and a centrally concentrated bulge. We assume that the stellar orbits, when projected onto the disc plane, are circular. The DF for the stars can then be written

$$
f(\mathbf{r}, \mathbf{v}, t)=\delta\left(v_{R}\right) \delta\left(v_{\phi}-R \Omega(R)\right) F\left(R, \phi, z, v_{z}, t\right),
$$

where $\Omega(R)=v_{c}(R) / R, \delta(\cdot)$ is the Dirac delta function, and $F\left(R, \phi, z, v_{z}, t\right)$ is the reduced DF. Upon integration over $v_{R}$ and $v_{\phi}$, the collisionless Boltzmann equation becomes

$$
\frac{\partial F}{\partial t}+\Omega(R) \frac{\partial F}{\partial \phi}+v_{z} \frac{\partial F}{\partial z}=\frac{\partial \psi}{\partial z} \frac{\partial F}{\partial v_{z}},
$$

where $\psi$ is the gravitational potential.

The vertical displacement of the disc is found by taking the $z$-moment of the DF:

$$
Z(R, \phi, t)=\int z F\left(R, \phi, z, v_{z}, t\right) d z d v_{z} .
$$

This quantity is essentially the analog of $Z$ in equation (6) for the thin, cold disc. Likewise, the vertical motion of the disc is

$$
V(R, \phi, t)=\int v_{z} F\left(R, \phi, z, v_{z}, t\right) d z d v_{z} .
$$

Multiply equation (17) by either $z$ or $v_{z}$ and integrate over $z$ and $v_{z}$ and we obtain

$$
\left(\frac{\partial}{\partial t}+\Omega(R) \frac{\partial}{\partial \phi}\right) Z=V
$$

and

$$
\left(\frac{\partial}{\partial t}+\Omega(R) \frac{\partial}{\partial \phi}\right) V=-\frac{\partial \psi}{\partial z}=F_{b}+F_{h}+F_{d}
$$

where in the last line, we've written out the separate contributions to the vertical force from the bulge, halo, and disc. Together, these equations lead to the standard second order equation for $Z$, which was first introduced in Hunter \& Toomre (1969).

For definiteness, we assume that the potential from the bulge and halo are the same as in the GALACTICs model described in Section 2. Near $z=0$, the contributions to the vertical restoring force due to these components are given, to a good approximation, by

$$
F_{b}(\mathbf{R}, t)=-v_{b}^{2} Z(\mathbf{R}, t) \quad F_{h}(\mathbf{R}, t)=-v_{h}^{2} Z(\mathbf{R}, t),
$$

where $\mathbf{R}=(R, \phi)$ in cylindrical coordinates. The vertical force due to the disc, to first order in $Z / R$, is

$$
\mathbf{F}_{d}=-G \int d \mathbf{R}^{\prime} \Sigma\left(R^{\prime}\right) \frac{Z(\mathbf{R}, t)-Z\left(\mathbf{R}^{\prime}, t\right)}{\left(R^{2}+R^{\prime 2}-2 R R^{\prime} \cos \varphi+z_{0}^{2}\right)^{3 / 2}},
$$

where $\varphi \equiv \phi-\phi^{\prime}$ and the "softening" parameter $z_{0}$ is introduced to make the integral regular at $\mathbf{R}=\mathbf{R}^{\prime}$ (Sparke \& Casertano 1988). (See Hunter \& Toomre 1969, Sparke \& Casertano 1988, and Nelson \& Tremaine 1995 for alternative ways of handling the $\mathbf{R}=\mathbf{R}^{\prime}$ singularity in the integrand.) Note that the term on the right-hand side that is proportional to $Z(\mathbf{R}, t)$ may be written $F_{1}=-v_{d}^{2} Z$, where

$$
v_{d}^{2}=G \int_{0}^{\infty} d R^{\prime} R^{\prime} \Sigma\left(R^{\prime}\right) H\left(R, R^{\prime}\right)
$$

and

$$
H\left(R, R^{\prime}\right)=\int_{0}^{2 \pi} \frac{d \varphi}{\left(R^{2}+R^{\prime 2}-2 R R^{\prime} \cos \varphi+z_{0}^{2}\right)^{3 / 2}}
$$




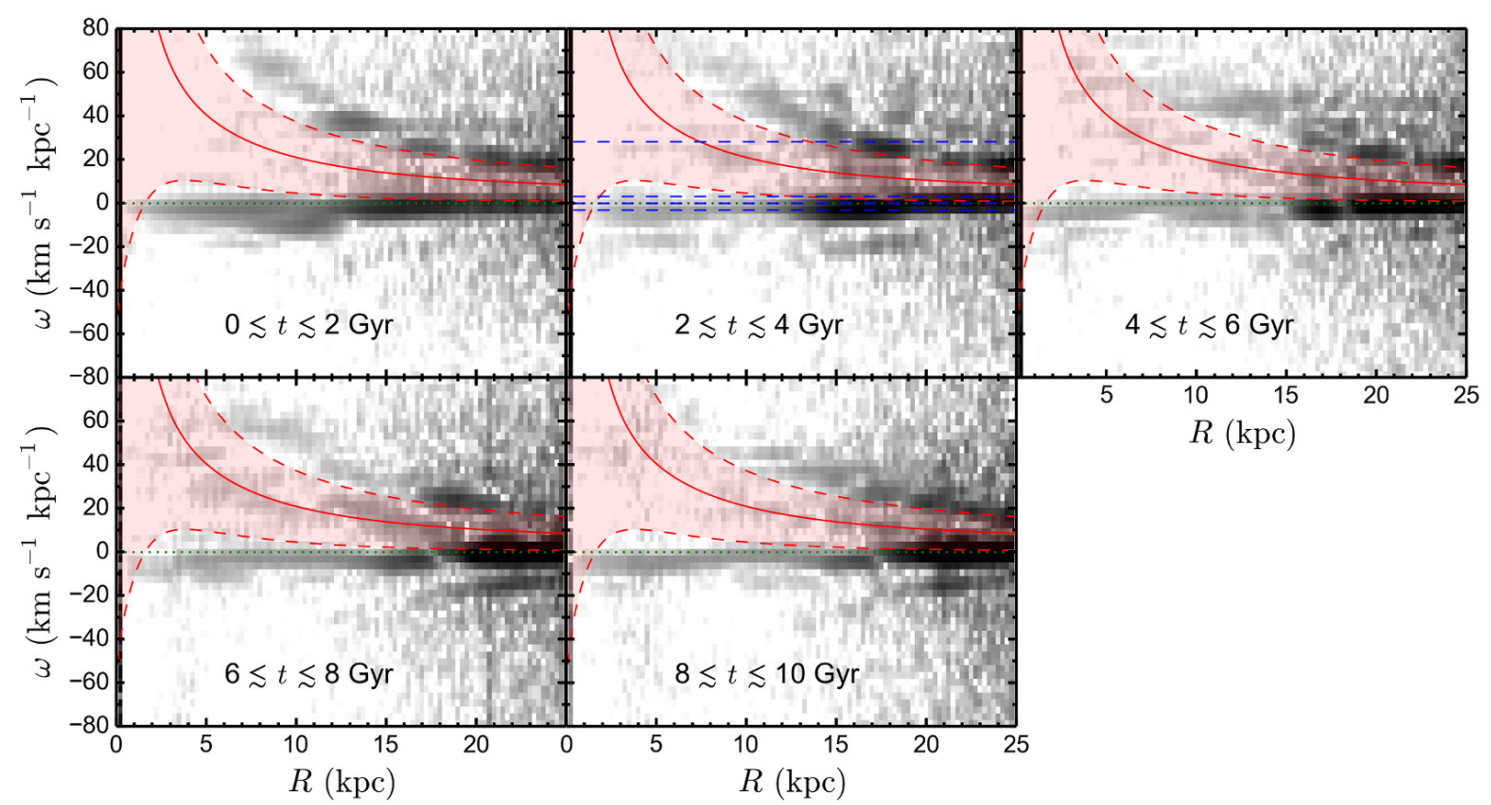

Figure 6. Two-sided frequency power spectra of $m=1$ bending waves for various $\sim 2$ Gyr time intervals, as indicated, for Model 1 . The scale of power is logarithmic and is constant across all time intervals shown. Overlaid on all panels in red are total circular frequency (solid) and vertical resonance (dashed) curves, $\omega=\Omega \pm v_{x}$, where $v_{x}=\sqrt{v_{b}^{2}+v_{h}^{2}}$ is the vertical forcing frequency from the bulge and halo, for the equilibrium initial conditions. The shaded red indicates the "forbidden" region between the resonances according to the WKB approximation (see Section 6.6.1 of Binney \& Tremaine 2008 and Section 4). The horizontal dotted green line references zero frequency. The horizontal dashed blue lines in the middle top panel indicate the frequencies considered in Fig. 7.

This term describes the vertical restoring force due to the equilibrium disc that acts on an element of the disc displaced from the midplane. We can then write

$$
v_{t}^{2}=v_{d}^{2}+v_{b}^{2}+v_{h}^{2},
$$

where $v_{t}$ is the total vertical epicyclic frequency. The term in equation (23) that is proportional to $Z\left(\mathbf{R}^{\prime}, t\right)$ describes the perturbing force on an unperturbed element in the disc due to those parts of the disc that have been displaced from the midplane.

For small displacements, we can write $Z$ and $V$ as superpositions of linear modes with $m$-fold azimuthal symmetry so that each mode has the form

$$
Z(R, \phi, t)=\operatorname{Re}\left\{\mathcal{Z}(R, \omega) e^{i(m \phi-\omega t)}\right\}
$$

and

$$
V(R, \phi, t)=\operatorname{Re}\left\{\mathcal{V}(R, \omega) e^{i(m \phi-\omega t)}\right\} .
$$

We then have

$$
-i(\omega-m \Omega) \mathcal{Z}(R, \omega)=\mathcal{V}(R, \omega)
$$

and

$$
\begin{aligned}
-i(\omega-m \Omega) & \mathcal{V}(R, \omega)=-v_{t}^{2} \mathcal{Z}(R, \omega) \\
& +G \int_{0}^{\infty} d R^{\prime} R^{\prime} \Sigma\left(R^{\prime}\right) \mathcal{Z}\left(R^{\prime}, \omega\right) I_{m}\left(R, R^{\prime}\right),
\end{aligned}
$$

where

$$
I_{m}\left(R, R^{\prime}\right)=\int_{0}^{2 \pi} \frac{d \varphi \cos m \varphi}{\left(R^{2}+R^{\prime 2}-2 R R^{\prime} \cos \varphi+z_{0}^{2}\right)^{3 / 2}}
$$

\subsection{Linear Ring Model and the WKB Approximation}

Following Sparke (1984) and Sparke \& Casertano (1988), we divide the disc into $\mathcal{N}$ concentric rings. Equations (29) and (30) together constitute an eigenvalue equation for the $2 \mathcal{N}$-dimensional vector $(\mathcal{Z}, \mathcal{V})^{T}$. The eigenvalues $\omega_{i} \quad(i=$ $1,2 \mathcal{N})$ are all real. We find that each eigenmode departs significantly from zero over a relatively narrow range in $R$. This point is illustrated in Fig. 8 for the case $m=1$ where we plot a horizontal line segment across the range in $R$ where the amplitude of the eigenmode exceeds $10 \%$ of its maximum value. The parameters for the $\mathcal{N}$-ring disc and the external potential of the bulge and halo are chosen to match our simulated Model 1.

Evidently, there are two continuous branches of eigenmodes. The patterns of modes along the upper- $\omega$ branch rotate in the same sense as the disc while those modes along the lower branch are counter-rotating or rotating at very small (nearly zero) positive frequency. In general, a disturbance localized in $R$ will involve a superposition of modes from the two branches. The part of the wave associated with modes from the upper branch, where $\omega$ is a decreasing function of $R$, will shear into a trailing spiral while the part asso- 

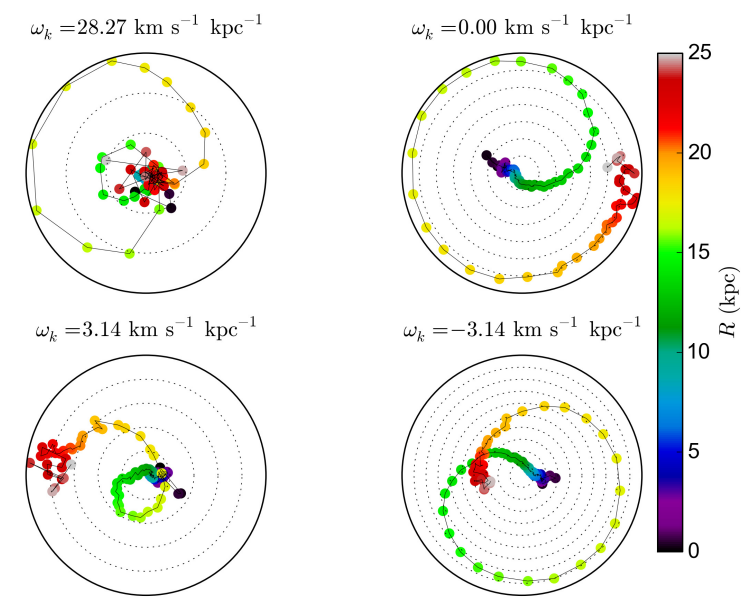

Figure 7. A form of tip-LON plots (so-called "Briggs" plots, see Briggs 1990) for four wave frequencies within the time interval $2<t<4$ Gyr (indicated in Fig. 6, top middle panel). For each frequency the disc is divided into concentric rings in radius. Each point in the figure corresponds to a radial ring, with the radius indicated by colour. The radial coordinate is the tilt, in degrees, of that ring, and the azimuthal coordinate is the position of maximum vertical displacement. Concentric dotted circles in the radial direction indicate tilt increments of $5^{\circ}$. High-frequency waves are morphologically trailing since the azimuth decreases with increasing radius. In contrast, waves near zero frequency are leading.

ciated with the lower branch will shear into a leading spiral. Since the magnitude of the differential rotation rate $|d \omega / d R|$ is smaller along the lower branch, phase mixing occurs more slowly for this part of the wave. Thus, over time we expect more pronounced leading waves of vertical oscillation, which is indeed what we find in the simulations (cf. Figs. 3 and 4).

For wavelengths that are small as compared with the scale length of the disc we may use a WKB approximation. The wavefronts are assumed to be perpendicular to the radial direction and the dispersion relation becomes (Hunter \& Toomre 1969; Sparke \& Casertano 1988; Nelson \& Tremaine 1995)

$$
(\omega-m \Omega(R))^{2}-4 \pi^{2} G \Sigma(R) / \lambda-v_{h}^{2}-v_{b}^{2}=0,
$$

where $\lambda$ is the wavelength of the perturbation. Corotation occurs when $\omega=\Omega$, while vertical resonances (the analogs of Linblad resonances) occur when $\omega=\Omega \pm v_{x}$ where $v_{x}=$ $\sqrt{v_{b}^{2}+v_{h}^{2}}$ is the vertical oscillation frequency associated with the halo and bulge (i.e., the fixed spheroidal components). From equation (32), we see that waves are excluded from the region in frequency space between these two resonances, the so-called "forbidden" region (Nelson \& Tremaine 1995; Binney \& Tremaine 2008, Section 6.6.1).

In addition to the results of the eigenmode analysis (Sparke \& Casertano 1988) in Fig. 8, we also show the corotation and vertical resonance curves as calculated in the WKB approximation. Indeed, the linear eigenmodes tend to lie along the two vertical resonances.

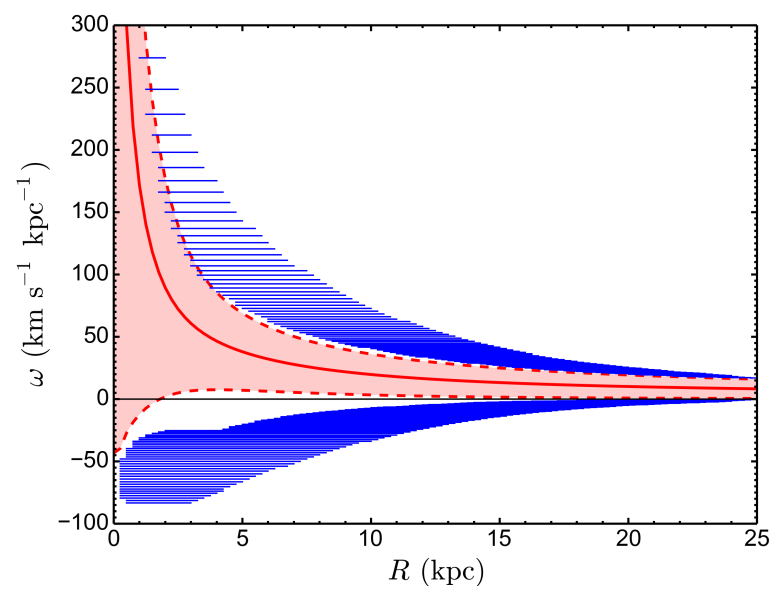

Figure 8. Frequency as a function of radius for $m=1$ bending wave eigenmodes of the linear ring model and the WKB analysis of Model 1 (see Section 4.2). The horizontal solid black line references zero frequency. Horizontal solid blue lines show the range in radius where the amplitude of each frequency eigenmode exceeds $10 \%$ of its maximum value. The solid red curve corresponds to the total circular frequency curve of the linear model, $\Omega$. Dashed red illustrates the $m=1$ vertical resonances, $\omega=\Omega \pm v_{x}$. Shaded red indicates the "forbidden" region between the two resonances, as predicted by the WKB approximation. Evidently, the linear eigenmodes lie outside of the forbidden region and tend to lie along the two vertical resonances.

\section{CORRUGATIONS IN EXTERNAL GALAXIES}

If warps and corrugations in Milky Way-like disc galaxies are easily excited and long lived then they should be observable in similar external galaxies. As shown in this work, and demonstrated in others (for example, see Gómez et al. 2017), spatial corrugations in the vertical direction are coupled to corrugations in stellar vertical velocity. Furthermore, Gómez et al. (2017) showed that vertical perturbations in simulated disc galaxies manifest in both the gaseous and stellar components. Thus, velocity corrugations should be observable in the line-of-sight kinematics of nearly face-on external disc galaxies. Integral field spectroscopy surveys such as Calar Alto Legacy Integral Field Area (CALIFA; Sánchez et al. 2012, but also see Falcón-Barroso et al. 2017), Mapping Nearby Galaxies at APO (MaNGA; Bundy et al. 2015), and DiskMass (Bershady et al. 2010) hold great promise for detecting bending waves in external galaxies since they gather spatially resolved information on both the stellar and gaseous components. It is most likely easier to detect any corrugations in the gaseous disc rather than the stellar component due to larger velocity dispersions in the latter. In this Section we outline a number of requirements needed to observe extragalactic bending waves and corrugations with respect to our simulations.

In Fig. 9 we show line-of-sight velocity maps for our Model 1 as viewed face-on and at inclinations $i=4^{\circ}, 8^{\circ}$, and $12^{\circ}$. Contributions to the line-of-sight velocity from both radial and azimuthal flows increase with increasing inclination. Nevertheless, hints of corrugation are still visible even at $i=12^{\circ}$. In principle, one might attempt to incorporate 
vertical motions into a model for two-dimensional velocity maps of spiral galaxies, as is done for non-circular flows in the presence of a bar (Spekkens \& Sellwood 2007; Sellwood \& Sánchez 2010; Sellwood \& Spekkens 2015).

We may thus ask, at what inclination do the motions in the vertical direction dominate the in-plane flows along the line-of-sight? That is, how face-on does a galaxy need to be? In Fig. 10 we consider the radial, azimuthal, and vertical bulk motions for Model 1. In particular, we show a time sequence of $m=1$ and $m=2$ Fourier mode amplitudes as a function of radius (i.e. equations 10 and 11, but for velocity rather than vertical displacement). In the inner disc, the largest amplitudes correspond to the $m=1$ and $m=2$ radial and azimuthal velocities, and are due to the bar. On the other hand, large $m=1$ vertical velocity amplitudes in the outer disc, especially at late times, are due to the warp and associated corrugations.

For an inclined disc, the $m=1$ and $m=2$ contributions, as well as higher $m$ terms from lower amplitude waves, mix into the line-of-sight velocity field. After accounting for the systematic velocity of a galaxy, the line-of-sight velocity can be decomposed as

$$
v_{\text {los }}=\left(v_{R} \sin \theta+v_{\phi} \cos \theta\right) \sin i+v_{z} \cos i,
$$

where $v_{R}, v_{\phi}$, and $v_{z}$ are Fourier expansions of each respective velocity component, $\theta$ is the position angle of the disc, and $i$ is the inclination. In Fig. 10, non-circular radial and azimuthal flows roughly equal that of the $m=1$ vertical component in the outer disc. Thus, upon averaging over the position angle term on the interval $[0, \pi]$, bulk $m=1$ vertical motions in the outer disc will be comparable to the integrated $m=1$ and $m=2$ in-plane contributions along the line-of-sight for an inclination of $\sim 38^{\circ}$. For inclinations of $\sim 20^{\circ}$ and $10^{\circ}$, the average vertical contribution to the line-of-sight velocity field would be roughly double and four times that, respectively, of the non-circular in-plane flows.

In addition to an inclination constraint, a very large field of view is needed in order to resolve the velocity corrugations that we find in the outer disc. The $m=1$ bending waves for Model 1 (Fig. 10) dominate in the region $R \sim 20-25 \mathrm{kpc}$, which corresponds to $\sim 6-8$ disc scale lengths, or $\sim 4-5$ effective radii (assuming an exponential disc and $1 R_{e} \simeq 1.7 R_{d}$ ). Furthermore, the spatial resolution required to resolve corrugations similar to those presented in this work should be at least $\sim 2-3 \mathrm{kpc}$.

The presence of strong spiral arms may complicate the interpretation of any observed "vertical motions". Since stars on the side of a face-on disc furthest from an observer are obscured by dust, compression and rarefaction of the disc perpendicular to the disc plane will appear as bulk vertical motions. Spiral arms can generate such breathing wave motions in the disc, and even arms with modest density contrasts can produce bulk motions comparable to what we observe in this work (Debattista 2014; Faure et al. 2014; Monari et al. 2016a,b). It could be that the corrugations observed by Sánchez-Gil et al. (2015) using long slit spectroscopy are associated with breathing mode perturbations from the spiral structure. However, the vertical motions will trace the spiral arms and follow a distinct compression and rarefaction pattern on either side of the arms. The bending waves we observe in our simulations are tightly wound and
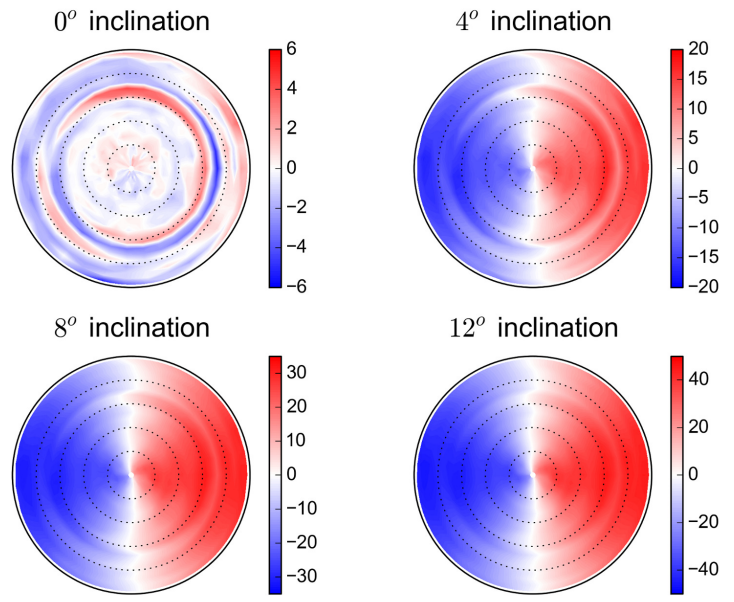

Figure 9. Projected line-of-sight velocity field of Model 1 at $t \sim 3.5 \mathrm{Gyr}$ for four inclinations, as indicated in the figure, rotated about the horizontal axis. Dotted concentric circles indicate increments of $5 \mathrm{kpc}$ in projected radius. The rotation of the disc is counter-clockwise in the face-on reference frame. The colour scale is in units of $\mathrm{km} \mathrm{s}^{-1}$, and differs in each panel to highlight the change in amplitude. Contributions from both radial and azimuthal flows become greater along the line-of-sight as the inclination increases. Despite this, hints of corrugated patterns are still apparent (for example, for $R$ between $\sim 15-20 \mathrm{kpc}$ ), even at the largest inclination shown in the figure.

leading, and therefore should be distinguishable from bulk motions induced by (trailing) spiral arms.

\section{DISCUSSION}

A satellite or dark matter subhalo that passes through a stellar disc will set up disturbances in the disc, which include vertical bending and breathing waves. The simulations presented in this paper demonstrate that bending waves also arise in an isolated Milky Way-like stellar disc without provocation from an outside agent such as a passing satellite.

At intermediate radii $(R \sim 8-15 \mathrm{kpc}$ for a Milky Waylike galaxy), these waves develop into tightly wound leading spirals with an amplitude and radial wavelength that is similar to those recently discovered in the Milky Way (Xu et al. 2015). In contrast to the hypothesis of Xu et al. (2015) that the corrugated structures are associated with spiral arms, we find that they are independent, both in morphology and pattern speed.

The bending waves in our simulations persist for many billions of years. Over time, bending wave power migrates outward. The warp begins at $R \sim 10-15 \mathrm{kpc}$ with an amplitude of $\sim 200 \mathrm{pc}$ that increases to $\sim 300-500 \mathrm{pc}$ closer to the disc's edge. These amplitudes are in agreement with recent measurements of the stellar warp in the Milky Way (see for example Reylé et al. 2009, and references therein). The outward migration of power is more dramatic for Model 1 where the bar and/or more massive disc plays a dominant role in suppressing bending waves, especially at intermediate radii. Since our Galaxy has a central bar, the implication is that 


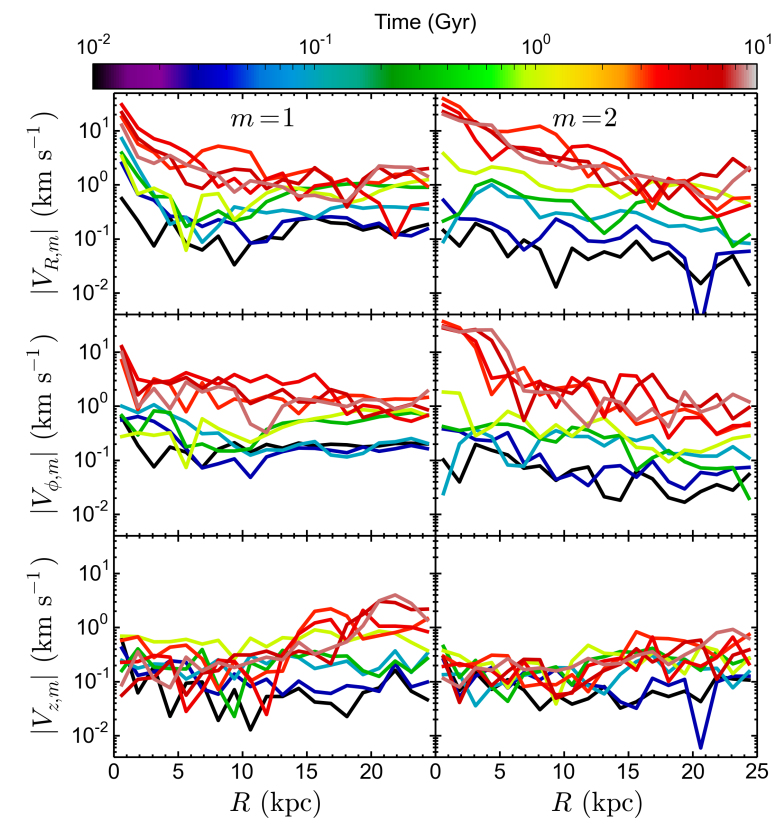

Figure 10. Time evolution of the $m=1$ and $m=2$ (left and right columns, respectively) Fourier coefficient magnitude as function of radius for cylindrical radial, azimuthal, and vertical velocities (top, middle, and bottom rows, respectively), for Model 1. Colour indicates the epoch of each magnitude's profile. The large in-plane $m=2$ amplitudes in the central disc are due to the bar, while the large $m=1$ amplitude of vertical velocity in the outer disc at later times is a manifestation of the warp and other vertical corrugation patterns. As the disc is projected onto the sky and inclined the $m=1,2$, and higher order terms (not shown) mix into the lineof-sight velocity. In the text we discuss at what inclinations the $m=1$ vertical motions dominate the mixed non-circular in-plane motions in the line-of-sight velocity field.

bending wave corrugations are efficiently damped in the inner disc, and should only be detected at Galactocentric radii greater than that of the Sun.

What then is the origin of the bending waves in our simulated galaxies? A likely culprit is the random noise of the halo and bulge particle distributions. To test this hypothesis, we re-simulated Model 1 with a fixed analytic potential for the bulge and halo and found that the vertical perturbations were negligible even though the disc developed spiral structure and a bar. We note bar formation was delayed as compared with the live halo case (see Sellwood 2016). As a further check, we evolved the live disc in a fixed potential that was generated by halo and bulge particles frozen in their initial positions. In this case, the disc developed a strong corrugation pattern of bending waves at intermediate radii and a large warp near the edge of the disc. Evidently, large-scale vertical waves can be excited by particle noise in the halo but not by structures in the disc itself, such as a bar or spiral arms.

Once formed, the evolution of bending waves is remarkably well described by linear perturbation theory, especially during the early stages of the simulations. The linear eigenmodes of a razor thin, dynamically cold disc form a continuum of bending waves, rather than discrete modes, with rotational frequencies that lie along the vertical resonance curves at $\omega=\Omega \pm v_{x}$ (Hunter \& Toomre 1969; Sparke \& Casertano 1988). Of course, our $N$-body discs have a nonzero vertical velocity dispersion and thickness. Also, the subsequent evolution of our live models obviously differs from that in the linear regime, where dynamical friction from the halo tends to efficiently damp bending waves on very short time-scales (Nelson \& Tremaine 1995). Furthermore, as the disc evolves, in-plane and vertical (time-dependent) inhomogeneities and asymmetries emerge, driven by the bar, spiral structure, mass redistribution within the disc, and resolution effects, which the linear theory and WKB analysis does not incorporate. Nevertheless, power in the $R-\omega$ plane concentrates along the two resonances and lies, for the most part, outside the region between them (i.e., the forbidden region as predicted by WKB theory).

Since both linear theory and the WKB analysis include self-gravity of the perturbations, we conclude that the phenomena observed in our discs are true bending waves. This contrasts with kinematical phase-wrapping explanations of the observed local bending and breathing modes in the Solar Neighbourhood as suggested by de la Vega et al. (2015). However, de la Vega et al. (2015) studied bending and breathing waves that arose from satellite interactions with the disc and close flybys of a fairly massive Sagittarius dwarf model. Admittedly, we do not consider direct interactions between the disc and external perturbing agents in this paper. One could imagine a two-stage scenario in which the waves present at the early stages of a satellite encounter behave at least somewhat kinematically. Later on, when these perturbations have phase-mixed and sheared into extended arcs they can be described by a ring model, in which selfgravity is a key ingredient.

We also conclude that our bending waves are not strongly Landau-damped, as one might have anticipated from the analysis of vertical oscillations in one-dimensional systems (Mathur 1990; Weinberg 1991; Louis 1992; Widrow \& Bonner 2015). Likewise, the waves are not strongly damped by dynamical friction due to the halo. If anything, a live halo (and bulge) appear to give rise to more vigorous perturbations perpendicular to the disc as predicted by Bertin \& Mark (1980) and in discord with the analysis of Nelson \& Tremaine (1995).

Our simulations are consistent with the picture presented in Binney et al. (1998). Recall that the authors of that paper simulated a disc-halo system where the disc was represented by rigid concentric rings while the halo constituted standard $N$-body particles. They showed that if the disc was initialized in the configuration of a discrete warp mode (e.g., the modified-tilt mode of Sparke \& Casertano 1988) the warp rapidly winds up while retaining its warp energy. They conclude that the true modes of the disc-halo system are different from those of the disc-static halo system, essentially because the halo responds to the warp and thus changes the potential in which the disc evolves. The spectral analysis of our simulations suggests that while the modes of the disc-halo system might differ quantitatively from those of the disk-static halo system, the general structure of the modes, at least those of the continuum, are qualitatively similar. The key point is that there exists a low-frequency branch where the differential precession is small at larger radii. Thus, the winding problem is alleviated and bending waves persist for many dynamical times. 
One might expect, then, that waves at smaller radii would be sheared out via differential rotation. The survival of these waves in our simulations, especially those along the low-frequency branch, is likely attributed to the strong selfgravity of the inner disc. Moreover, random in-plane motions act to further stiffen the inner disc and resist bending (Debattista \& Sellwood 1999). A straight LON and constant pattern speed in the inner disc indicates that the waves rotate coherently and cohesively, connecting the global structure of low-frequency waves between the inner and outer disc. The waves in the inner disc of our simulations differ from that predicted by the linear ring model since neighbouring rings cannot be considered to oscillate independently and epicyclic motions are not taken into account. This point implies at least some degree of non-linearity present in our simulations regarding the bending waves - the waves we see may very well be akin to the true modes of the disc hinted at by Binney et al. (1998).

\section{CONCLUSIONS}

Our main conclusion is that bending waves should be generic, long-lived features of Milky Way-like disc galaxies irrespective of whether the disc has been perturbed by a passing satellite or dark matter subhalo. This conclusion is based on the results from N-body simulations of isolated Milky Way-like galaxies. In particular, we focus on two models: one with a maximal bar-forming disc and the other with a submaximal disc which only forms flocculent spiral structure. In both models, bending waves develop across the entire disc within the first billion years of the simulation. At intermediate radii the waves manifest as leading, tightly wound corrugations, which extend over a large range in azimuth and match smoothly on to the warp that develops at the edge of the disc.

A major goal of this paper is to develop tools for the study of bending waves in simulations and observations. Our approach is based on the spectral analysis of surface density maps for simulated disc galaxies - a tool that has proved indispensable for understanding the dynamics of bars and spiral structure. In principle, the complete dynamical state of a stellar disc is encapsulated in the DF, a complicated function of the six phase space coordinates and time. To simplify the analysis, one can derive a surface density map, essentially by integrating the DF over $\mathbf{v}$ and $z$. The standard procedure is to then divide the disc into radial bins and write the surface density in each bin as a series of functions with $m$-fold azimuthal symmetry. The coefficients provide the relative strength of the different angular modes as a function of $R$ and $t$. By Fourier transforming in time, we can also obtain a power spectrum in $R$ and $\omega$.

For this work, we begin with the $z$ and $v_{z}$ moments of the DF across the disc, which provide a measure of bending waves in the disc. As with surface density maps, we can decompose maps of $\langle z\rangle$ and $\left\langle v_{z}\right\rangle$ as Fourier series in $\phi$. We focus on the $m=1$ waves, which is the dominant term not only for the warp but also for the bending waves that occur at intermediate radii. Furthermore, the $m=1$ waves provide a direct link to tilted ring models for warped galaxies and the eigenfunction calculations of Hunter \& Toomre (1969) and Sparke \& Casertano (1988). From Fig. 5, we conclude that at a given $R$, the amplitude of the $m=1$ bending waves grows with time until reaching some maximal value, after which it remains roughly constant. The maximum amplitude is an increasing function in $R$ as is the time at which it is reached. The upshot is that over time, the strength of the bending waves near the edge of the disc increases relative to that of waves in the inner part of the disc.

The long-lived nature of bending waves is consistent with the conclusions of Binney et al. (1998), who showed that a live halo responds to vertical displacements on a relatively short time-scale. In essence, bending waves are a phenomena of the disc-halo system. In the absence of this coupling between the disc and live halo, dynamical friction would efficiently damp the bending waves, as was suggested by Nelson \& Tremaine (1995).

The connection between linear theory, in the form of either eigenfunction or WKB analyses, and the simulation results is most clearly seen in Figs. 6 and 8. The agreement is impressive, as power in the $m=1$ waves tends to reside just outside the WKB-forbidden region between the two vertical resonance curves. At early times, the power is distributed approximately uniformly throughout the disc while at late times power is concentrated near the outer edge of the disc, lending credence to an inside-out formation scenario of the warp. We emphasize that central to the linear theory calculations is a gravitational restoring force that acts on perturbations in the disc. Hence, the agreement between simulations and linear theory bolsters our claim that we are observing (at least in the simulations) bona fide gravity waves.

It was a surprise (at least to us) that bending waves arose in a disc embedded in a smooth dark matter halo. Evidently, particle noise in the halo is enough to provoke vertical waves in a stellar disc. Of course, dark matter haloes in $\Lambda \mathrm{CDM}$ cosmologies are predicted to host a system of satellite galaxies and dark matter subhaloes (for example, see the early works of Klypin et al. 1999 and Moore et al. 1999). In a realistic galaxy, a small fraction of these halo objects will pass through the disc, an interaction that is characterized by a series of phases. In the first, a local region of the disc is perturbed by the passing object. The response of individual stars will depend on how well their vertical epicyclic motions match the time-dependence of the gravitational field (Sellwood et al. 1998; Widrow et al. 2014). The localized perturbation is then sheared out due to differential rotation and phase mixing (Widrow et al. 2014; de la Vega et al. 2015) but soon begins to behave as a tightly wound bending wave with gravity providing the restoring force. In essence, this is the phase investigated here. Eventually, the wave energy is dissipated through further phase mixing and Landau damping. Consequently, the disc then becomes a little thicker and kinematically hotter. Using the shot noise in our simulations as a proxy for asymmetries in haloes, it is tangible to imagine bending waves in Milky Way-like (stellar) discs to be continually excited.

One of the goals of the Gaia observing mission (Perryman et al. 2001; Gaia Collaboration et al. 2016a, but see Gaia Collaboration et al. 2016b for an overview of DR1) is to provide a map of the mean velocity field over a substantial fraction of the Galactic disc. The picture presented here suggests that this map will reveal a mix of localized flows and larger scale motions that connect waves at intermediate radii with the outer warp (see, for example, Abedi et al. 
2014 regarding Gaia and the Galactic warp). In principle, the former will tell us something about perturbers roaming the halo while the latter may say more about the structure of the disc. We predict that less dispersive low-frequency $m=1$ bending waves, which manifest as tightly wound and leading radially dependent vertical asymmetries in position and velocity, should be prevalent at radii just beyond the Sun. Although similar features have already been observed in the Milky Way's density distribution (Xu et al. 2015), the key will be testing the true wave nature of these patterns using proper motion and radial velocity measurements from the next Gaia data release. In theory, the analysis tools based on moments in $z$ and $v_{z}$, and azimuthal Fourier modes, can be applied to Gaia data, though there, one must contend with selection effects, observational uncertainties, and obscuration due to dust.

One possible complication, not discussed here, is that different components of the disc may respond differently to a passing satellite. Bovy \& Rix (2013) have suggested that to do an Oort type analysis (that is, to infer the surface density, vertical force, and density of dark matter in the disc) one should divide disc stars into bins based on $[\alpha / \mathrm{Fe}]$ and $[\mathrm{Fe} / \mathrm{H}]$ - the idea being that the stars in each bin can be treated as a distinct isothermal tracer of the gravitational potential. On the other hand, if the disc is in a continual state of disequilibrium, and if the manifestation of disequilibrium varies from one population to the next, then populationdependent systematic errors might creep into this sort of analysis (Banik et al. 2017). Put another way, discrepancies in the inferred potential might signal that the Galaxy is in a perturbed state.

In summary, Gaia may well find complicated patterns in the bulk motions of disc stars. The challenge will then be to characterize these motions, perhaps with the spectral methods described here, and disentangle the effects of external perturbers and internal dynamics.

\section{ACKNOWLEDGEMENTS}

The authors would like to thank the anonymous referee for helpful comments that improved the quality of the manuscript. The authors are also grateful to Heidi Newberg, Kristine Spekkens, and Sebastián Sánchez for useful conversations, and Jacob Bauer for his assistance with the simulations. We also acknowledge the use of computational resources at the Centre for Advanced Computing. This work was supported by the Natural Sciences and Engineering Research Council of Canada through the Postgraduate Scholarship Program (MHC) and Discovery Grant Program (LMW).

\section{REFERENCES}

Abedi H., Mateu C., Aguilar L. A., Figueras F., Romero-Gómez M., 2014, MNRAS, 442, 3627

Alfaro E. J., Pérez E., González Delgado R. M., Martos M. A., Franco J., 2001, ApJ, 550, 253

Banik N., Widrow L. M., Dodelson S., 2017, MNRAS, 464, 3775

Battaner E., Florido E., Sanchez-Saavedra M. L., 1990, A\&A, 236,1
Bershady M. A., Verheijen M. A. W., Swaters R. A., Andersen D. R., Westfall K. B., Martinsson T., 2010, ApJ, 716, 198 Bertin G., Mark J. W.-K., 1980, A\&A, 88, 289

Binney J., 1992, ARA\&A, 30, 51

Binney J., Tremaine S., 2008, Galactic Dynamics: Second Edition. Princeton University Press

Binney J., Jiang I.-G., Dutta S., 1998, MNRAS, 297, 1237

Bizyaev D. V., Kautsch S. J., Sotnikova N. Y., Reshetnikov V. P., Mosenkov A. V., 2017, MNRAS, 465, 3784

Bottema R., 1993, A\&A, 275, 16

Bovy J., Rix H.-W., 2013, ApJ, 779, 115

Briggs F. H., 1990, ApJ, 352, 15

Bundy K., et al., 2015, ApJ, 798, 7

Carlin J. L., et al., 2013, ApJ, 777, L5

Chandrasekhar S., 1943, ApJ, 97, 255

Cox A. L., Sparke L. S., van Moorsel G., Shaw M., 1996, AJ, 111, 1505

D'Onghia E., Madau P., Vera-Ciro C., Quillen A., Hernquist L., 2016, ApJ, 823, 4

de la Vega A., Quillen A. C., Carlin J. L., Chakrabarti S., D'Onghia E., 2015, MNRAS, 454, 933

Debattista V. P., 2014, MNRAS, 443, L1

Debattista V. P., Sellwood J. A., 1999, ApJ, 513, L107

Dekel A., Shlosman I., 1983, in Athanassoula E., ed., IAU Symposium Vol. 100, Internal Kinematics and Dynamics of Galaxies. p. 187

Falcón-Barroso J., et al., 2017, A\&A, 597, A48

Faure C., Siebert A., Famaey B., 2014, MNRAS, 440, 2564

Feldmann R., Spolyar D., 2015, MNRAS, 446, 1000

Ferguson D., Gardner S., Yanny B., 2017, preprint, (arXiv: 1706.01900)

Gaia Collaboration et al., 2016a, A\&A, 595, A1

Gaia Collaboration et al., 2016b, A\&A, 595, A2

Gómez F. A., Minchev I., O'Shea B. W., Beers T. C., Bullock J. S., Purcell C. W., 2013, MNRAS, 429, 159

Gómez F. A., White S. D. M., Marinacci F., Slater C. T., Grand R. J. J., Springel V., Pakmor R., 2016, MNRAS, 456, 2779

Gómez F. A., White S. D. M., Grand R. J. J., Marinacci F., Springel V., Pakmor R., 2017, MNRAS, 465, 3446

Hammersley P. L., López-Corredoira M., 2011, A\&A, 527, A6

Hunter C., Toomre A., 1969, ApJ, 155, 747

Kahn F. D., Woltjer L., 1959, ApJ, 130, 705

Kazantzidis S., Bullock J. S., Zentner A. R., Kravtsov A. V., Moustakas L. A., 2008, ApJ, 688, 254

Klypin A., Kravtsov A. V., Valenzuela O., Prada F., 1999, ApJ, 522,82

Kuijken K., Dubinski J., 1995, MNRAS, 277, 1341

Levine E. S., Blitz L., Heiles C., 2006, ApJ, 643, 881

Li J., Newberg H. J., Carlin J. L., Deng L., Newby M., Willett B. A., Xu Y., Luo Z., 2012, ApJ, 757, 151

López-Corredoira M., Betancort-Rijo J., Beckman J. E., 2002, A\&A, 386, 169

Louis P. D., 1992, MNRAS, 258, 552

Lynden-Bell D., 1965, MNRAS, 129, 299

Martin N. F., Ibata R. A., Bellazzini M., Irwin M. J., Lewis G. F., Dehnen W., 2004, MNRAS, 348, 12

Martos M. A., Cox D. P., 1998, ApJ, 509, 703

Martos M., Allen C., Franco J., Kurtz S., 1999, ApJ, 526, L89

Mathur S. D., 1990, MNRAS, 243, 529

Momany Y., Zaggia S. R., Bonifacio P., Piotto G., De Angeli F., Bedin L. R., Carraro G., 2004, A\&A, 421, L29

Momany Y., Zaggia S., Gilmore G., Piotto G., Carraro G., Bedin L. R., de Angeli F., 2006, A\&A, 451, 515

Monari G., Famaey B., Siebert A., 2015, MNRAS, 452, 747

Monari G., Famaey B., Siebert A., 2016a, MNRAS, 457, 2569

Monari G., Famaey B., Siebert A., Grand R. J. J., Kawata D., Boily C., 2016b, MNRAS, 461, 3835 
Moore B., Ghigna S., Governato F., Lake G., Quinn T., Stadel J., Tozzi P., 1999, ApJ, 524, L19

Morganson E., et al., 2016, ApJ, 825, 140

Navarro J. F., Frenk C. S., White S. D. M., 1996, ApJ, 462, 563

Nelson R. W., Tremaine S., 1995, MNRAS, 275, 897

Newberg H. J., et al., 2002, ApJ, 569, 245

Peñarrubia J., et al., 2005, ApJ, 626, 128

Perryman M. A. C., et al., 2001, A\&A, 369, 339

Press W. H., Teukolsky S. A., Vetterling W. T., Flannery B. P., 2007, Numerical Recipes. The Art of Scientific Computing, 3rd edn. Cambridge University Press

Prugniel P., Simien F., 1997, A\&A, 321, 111

Purcell C. W., Bullock J. S., Tollerud E. J., Rocha M., Chakrabarti S., 2011, Nature, 477, 301

Revaz Y., Pfenniger D., 2001, A\&A, 372, 784

Revaz Y., Pfenniger D., 2004, A\&A, 425, 67

Reylé C., Marshall D. J., Robin A. C., Schultheis M., 2009, A\&A, 495, 819

Roškar R., Debattista V. P., Quinn T. R., Wadsley J., 2012, MNRAS, 426, 2089

Sánchez-Gil M. C., Alfaro E. J., Pérez E., 2015, MNRAS, 454, 3376

Sánchez S. F., et al., 2012, A\&A, 538, A8

Sellwood J. A., 2013, Dynamics of Disks and Warps. p. 923, doi:10.1007/978-94-007-5612-0_18

Sellwood J. A., 2016, ApJ, 819, 92

Sellwood J. A., Athanassoula E., 1986, MNRAS, 221, 195

Sellwood J. A., Sánchez R. Z., 2010, MNRAS, 404, 1733

Sellwood J. A., Spekkens K., 2015, preprint, (arXiv:1509.07120)

Sellwood J. A., Nelson R. W., Tremaine S., 1998, ApJ, 506, 590

Shen J., Sellwood J. A., 2006, MNRAS, 370, 2

Sparke L. S., 1984, ApJ, 280, 117

Sparke L. S., Casertano S., 1988, MNRAS, 234, 873

Spekkens K., Sellwood J. A., 2007, ApJ, 664, 204

Springel V., 2005, MNRAS, 364, 1105

Sun N.-C., et al., 2015, Research in Astronomy and Astrophysics, 15,1342

Terzić B., Graham A. W., 2005, MNRAS, 362, 197

Toomre A., 1964, ApJ, 139, 1217

Toomre A., 1983, in Athanassoula E., ed., IAU Symposium Vol. 100, Internal Kinematics and Dynamics of Galaxies. pp 177185

Weinberg M. D., 1991, ApJ, 373, 391

Widrow L. M., Bonner G., 2015, MNRAS, 450, 266

Widrow L. M., Pym B., Dubinski J., 2008, ApJ, 679, 1239

Widrow L. M., Gardner S., Yanny B., Dodelson S., Chen H.-Y., 2012, ApJ, 750, L41

Widrow L. M., Barber J., Chequers M. H., Cheng E., 2014, MNRAS, 440, 1971

Williams M. E. K., et al., 2013, MNRAS, 436, 101

Xu Y., Newberg H. J., Carlin J. L., Liu C., Deng L., Li J., Schönrich R., Yanny B., 2015, ApJ, 801, 105

Yanny B., Gardner S., 2013, ApJ, 777, 91

Yanny B., et al., 2003, ApJ, 588, 824

Younger J. D., Besla G., Cox T. J., Hernquist L., Robertson B., Willman B., 2008, ApJ, 676, L21

This paper has been typeset from a $\mathrm{T}_{\mathrm{E}} \mathrm{X} / \mathrm{LATEX}$ file prepared by the author. 